\title{
Genetic Manipulation of Non-tuberculosis Mycobacteria
}

\author{
Nyaradzai Mitchell Chimukuche and Monique J. Williams* \\ Department of Molecular and Cell Biology, University of Cape Town, Cape Town, South Africa
}

Non-tuberculosis mycobacteria (NTMs) comprise a large group of organisms that are phenotypically diverse. Analysis of the growing number of completed NTM genomes has revealed both significant intra-genus genetic diversity, and a high percentage of predicted genes that appear to be unique to this group. Most NTMs have not been studied, however, the rise in NTM infections in several countries has prompted increasing interest in these organisms. Mycobacterial research has recently benefitted from the development of new genetic tools and a growing number of studies describing the genetic manipulation of NTMs have now been reported. In this review, we discuss the use of both site-specific and random mutagenesis tools in NTMs, highlighting the challenges that exist in applying these techniques to this diverse group of organisms.

OPEN ACCESS

Edited by: Apichai Tuanyok, University of Florida, United States

Reviewed by: Joseph Oliver Falkinham, Virginia Tech, United States Jianping Xie, Southwest University, China Yukiko Nishiuchi,

Osaka City University, Japan

*Correspondence:

Monique J. Williams monique.williams@uct.ac.za

Specialty section:

This article was submitted to Evolutionary and Genomic Microbiology, a section of the journal

Frontiers in Microbiology

Received: 27 November 2020 Accepted: 27 January 2021 Published: 17 February 2021

Citation:

Chimukuche NM and Williams MJ (2021) Genetic Manipulation of

Non-tuberculosis Mycobacteria.

Front. Microbiol. 12:633510. doi: 10.3389/fmicb.2021.633510
Keywords: non-tuberculosis mycobacteria (NTMs), mutagenesis, homologous recombination, recombineering, transposon mutagenesis, CRISPR/Cas

\section{INTRODUCTION}

The genus Mycobacterium is a diverse group of organisms that includes more than 180 species. Species within the genus that do not cause tuberculosis (Mycobacterium tuberculosis complex) or leprosy (Mycobacterium leprae and Mycobacterium lepromatosis) are termed non-tuberculosis mycobacteria (NTMs) or environmental mycobacteria. While most of these species are nonpathogenic and occur ubiquitously in the environment, several are opportunistic pathogens causing infection of the lungs, soft tissue, and bones (Griffith et al., 2007).

Mycobacterial research has benefitted from two major advances in the last two decades. Firstly, improvements in next-generation sequencing technology has rapidly increased the number of completed whole genome sequences; currently more than 150 mycobacterial genomes are publicly available (Gupta et al., 2018). Secondly, the development of new genetic tools, particularly those for efficiently generating site-specific mutations in mycobacteria, has facilitated the study of gene function. Although in silico tools can provide some insight into gene function, these predictions require experimental validation (Niehaus et al., 2015) and rely on inferences from homologs. Analysis of 41 NTM genomes revealed that $61 \%$ of the predicted genes could not be assigned a function by genome annotation tools (Fedrizzi et al., 2017), highlighting the need for functional studies within NTMs. One of the major advantages of using site-specific mutagenesis to elucidate gene function is the ability to study gene products in their cellular context. In the case of clinically relevant NTMs, this is crucial for understanding NTM pathogenesis and disease (Russell et al., 2010), and for the development of more effect antibiotics to treat NTM infections (Wu et al., 2018).

Studying NTM physiology and gene function also has relevance for other diseases. Heat-killed Mycolicibacterium vaccae (SRL 172) has shown some potential in the treatment of prostate (Hrouda et al., 1998) and lung cancer (O'Brien et al., 2000), suggesting that NTMs could have utility as immunomodulators. This is consistent with the hypothesis that NTM-exposure influences BCG 
vaccine efficacy in preventing pulmonary tuberculosis in different populations (Fine, 1995). Apart from impacting human health, NTMs also cause disease in animals, and infection of ruminants by Mycobacterium avium ssp. paratuberculosis results in substantial losses for the livestock industry (Whittington et al., 2019). NTMs may also be useful for bioproduction; Mycolicibacterium smegmatis has been used as an alternative to Escherichia coli for recombinant protein production (Goldstone et al., 2008) while the large number of genes unique to mycobacteria represent an untapped resource for novel bio-transformations and small molecule production. The genetic manipulation of NTMs therefore has significance for several areas.

Herein, we review studies that have undertaken genetic manipulation in NTMs, discussing the advantages and disadvantages of each mutagenesis method and highlighting the challenges of applying these techniques to NTMs.

\section{ONE-STEP AND TWO-STEP ALLELIC EXCHANGE MUTAGENESIS}

Allelic exchange mutagenesis harnesses the DNA repair mechanism of homologous recombination (HR) to facilitate replacement of a region of chromosomal DNA. The process utilizes an allelic exchange substrate (AES), which is a piece of DNA that carries the desired mutation and regions of homology to the locus of interest. Following introduction of the AES into the cell, endogenous HR proteins catalyze recombination events such that the homologous region in the chromosome is replaced by the AES (McFadden, 1996). AESs are either suicide vectors, which have no origin of replication, or vectors that replicate under specific conditions, most commonly a specific temperature (Mycobacteria Protocols | SpringerLink) (Parish and Roberts, 2015). For one-step allelic exchange, homologous regions $(1,5-2 \mathrm{~kb})$ from either side of the desired deletion are cloned upstream and downstream of an antibiotic resistance marker in a vector backbone to create the AES. A marked deletion is generated when homologous recombination occurs between the AES and the chromosome at both the upstream and downstream regions (double cross-over), i.e., the sequence between the upstream and downstream homologous regions is replaced by the antibiotic resistance marker in the chromosome. Antibiotic selection facilitates isolation of the resulting marked mutants. In two step allelic exchange mutagenesis, AESs contain an additional negative selection marker that results in death of the bacteria during selection, while the antibiotic resistance marker can be positioned in the vector backbone to create unmarked deletions (Parish and Stoker, 2000). The first step of the process uses positive selection (usually antibiotic resistance) to isolate clones that have undergone a single homologous recombination event (single cross-over). This results in integration of the AES, creating a strain that carries both the wild-type and mutant alleles. In the second selection step, the second recombination event is selected for using negative selection, and results in removal of the vector backbone and one of the alleles. Removal of the wild-type allele results in the generation of a mutant strain, and vice versa.

The feasibility of using allelic exchange for mutagenesis in Mycobacterium intracellulare was initially demonstrated by showing that homologous recombination resulted in integration of a suicide vector in a site-specific manner (Marklund et al., 1995). One-step allelic exchange mutagenesis was subsequently employed to delete the 19-kilodalton antigen (19Ag) using a suicide vector (Table 1) (Mahenthiralingam et al., 1998). The vector was engineered to contain $6.2 \mathrm{~kb}$ of homology, in which the $19 \mathrm{Ag}$ gene had been disrupted by a gentamycin $(\mathrm{Gm})$ cassette. Electroporations performed with $1 \mu \mathrm{g}$ of the suicide vector and the $M$. intracellulare mouse-avirulent strain, TMC1403, yielded between 8 and $15 \mathrm{Gm}^{\mathrm{r}}$ colonies. Only one of these colonies was a marked mutant, indicative of the low efficiency of this method; the remaining colonies were either single cross-overs or had undergone non-homologous recombination. Attempts to use the same suicide vector in the mouse-virulent strain, D673, failed to yield any mutants. In contrast, a mutant derivative of D673, FM1, was amenable to mutagenesis by allelic exchange. The FM1 mutant displayed altered colony morphology, suggesting that it had a cell wall defect that increased its transformation efficiency. This study illustrates that because of low homologous recombination frequencies $\left(10^{-4}\right.$ to $\left.10^{-6}\right)$, the low transformation efficiency of NTMs can become a limiting factor in generating mutants.

One strategy to overcome the low transformation efficiency of electroporation is to use mycobacteriophages to deliver DNA. This involves incorporation of the AES (including selection/counter selection markers) into a shuttle phasmid. The most commonly used phasmid, phAE87, is a conditionally replicating derivative of phage TM4 which replicates at $30^{\circ} \mathrm{C}$ but not at $37^{\circ} \mathrm{C}$ (Bardarov et al., 2002). Following transduction, bacteria that have undergone recombination are isolated by incubation and selection at the non-permissive temperature $\left(37^{\circ} \mathrm{C}\right)$. Attempts to use the phAE87 transduction protocol developed for Mycobacterium bovis/M. tuberculosis in Mycobacterium avium subsp. paratuberculosis resulted in a high number of spontaneous mutants and very few allelic exchange mutants (Park et al., 2008). A significantly higher proportion of allelic exchange mutants [78-100\% of hygromycin (Hyg) resistant colonies] was obtained using a modified method, modifications that included removal of bacterial clumps prior to transduction and performing selection on a higher antibiotic concentration. Transduction and selection procedures therefore need to be optimized for each NTM species.

In mycobacteria, spontaneous resistance to antibiotics used for selection arises at a similar frequency to homologous recombination (Parish et al., 1999; Bardarov et al., 2002; Viljoen et al., 2018). This can be problematic during allelic exchange mutagenesis as it increases the number of colonies that need to be screened following selection. The emergence of spontaneous mutants is determined by a number of factors, including the number of changes that lead to the phenotype and the resulting fitness cost (Martinez and Baquero, 2000). Using a combination of selection markers or individual markers that develop spontaneous mutants at a lower frequency is 
therefore a means of overcoming this problem. A system developed for use in Mycobacteroides abscessus combined antibiotic selection with fluorescent selection by incorporating tdTomato or mWasabi in the suicide vector (Viljoen et al., 2018). This approach relies on one-step allelic exchange and integration of the suicide vector (single-cross over) into the target gene to disrupt its function. Screening of fluorescent colonies following selection revealed that homologous recombination had occurred in all cases, suggesting that the frequency of non-homologous (illegitimate) recombination in $M$. abscessus is lower than for M. smegmatis, M. bovis and M. tuberculosis. The length of the homology in the suicide vector impacted the frequency of recombination in $M$. abscessus, with regions below 500 bp yielding very few colonies. In $M$. avium, combining antibiotic selection with the $x y l E$ gene (E.C. 1.13.1.2) enabled identification of recombinant colonies by their yellow appearance in the presence of a catechol solution (Irani et al., 2004). Only $25-40 \%$ of the hygromycin colonies stained yellow, indicating a high rate of spontaneous hygromycin resistance in $M$. avium. An alternate strategy employed in $M$. avium was to use the Streptomyces coelicolor leuD gene as a selection marker to complement pre-existing leucine auxotrophy (Otero et al., 2003). This marker was chosen because reversion of leucine auxotrophy occurs at a very low frequency $\left(10^{-11}\right)$. Four of the seven colonies isolated on minimal media had undergone homologous recombination, while non-homologous recombination had occurred in the remaining three colonies.
Although this system overcomes the problem of spontaneous mutations, the need for a pre-existing auxotrophic strain limits its application.

The development of a two-step allelic exchange system by Parish and Stoker (2000) has significantly improved the efficiency with which mutations can be introduced in mycobacteria (Parish and Stoker, 2000). This system relies on both antibiotic resistance and $\beta$-galactosidase activity (lac Z) for positive selection of the first cross-over event, and sucrose sensitivity $(s a c B)$ for negative selection of the second cross-over event. Two-step allelic exchange using sucrose sensitivity as a counter selection marker has been used to generate mutants in several NTMs, including Mycobacterium marinum (Zhang et al., 2016), M. avium (Irani et al., 2004; Krzywinska et al., 2005) and Mycobacteroides chelonae (Moura et al., 2014) (see Table 1 for further details). The $s a c B$ marker does not work in M. abscessus (Medjahed and Reyrat, 2009), and therefore alternative counter-selection markers have been developed for this organism. The M. tuberculosis katG gene encodes the catalase-peroxidase responsible for converting isoniazid into an active form (Vilchèze and Jacobs, 2014). Inclusion of katG on two-step AESs sensitizes $M$. abscessus transformants to isoniazid, facilitating counter-selection on isoniazid (Rominski et al., 2017). Similarly, inclusion of the E. coli galK gene, which converts 2-deoxygalactose (2-DOG) to the toxic product 2-deoxygalactose-1-phosphate, enables counter selection on 2-DOG (Gregoire et al., 2017). Although twostep allelic exchange is more efficient, the process is slow and

TABLE 1 | Examples of one-step and two-step allelic exchange mutagenesis in non-tuberculosis mycobacteria

\begin{tabular}{|c|c|c|c|c|c|c|}
\hline Mycobacterial species & $\begin{array}{l}\text { DNA delivery } \\
\text { method }\end{array}$ & Target gene(s) & $\begin{array}{l}\text { Allelic exchange } \\
\text { substrate }\end{array}$ & $\begin{array}{l}\text { Positive selection } \\
\text { markers }\end{array}$ & $\begin{array}{l}\text { Negative } \\
\text { selection markers }\end{array}$ & References \\
\hline \multicolumn{7}{|l|}{ One-step allelic exchange } \\
\hline $\begin{array}{l}\text { M. intracellulare (TMC1403, } \\
\text { D673, FM1) }\end{array}$ & Electroporation & $19 \mathrm{Ag}$ & Suicide vector & $\mathrm{Gm}^{r}$ & $\mathrm{n} / \mathrm{a}$ & $\begin{array}{l}\text { Mahenthiralingam } \\
\text { et al. (1998) }\end{array}$ \\
\hline $\begin{array}{l}\text { M. avium subsp. } \\
\text { paratuberculosis (K10, } \\
\text { K10-GFP) }\end{array}$ & Transduction & relA pknG Isr2 & $\begin{array}{l}\text { Phasmid derived from } \\
\text { pHAE87 }\end{array}$ & $\mathrm{Km}^{r} \mathrm{Hyg}^{r}$ & $\mathrm{n} / \mathrm{a}$ & Park et al. (2008) \\
\hline $\begin{array}{l}\text { M. avium subsp. } \\
\text { paratuberculosis (K10) }\end{array}$ & Transduction & mce4 & $\begin{array}{l}\text { Phasmid derived from } \\
\text { pHAE87 }\end{array}$ & $\mathrm{Km}^{r}$ & $\mathrm{n} / \mathrm{a}$ & Alonso et al. (2020) \\
\hline $\begin{array}{l}\text { M. abscessus subsp. } \\
\text { abscessus (CIP104526) }\end{array}$ & Electroporation & $\begin{array}{l}m m p L 4 a \\
m m p S 4\end{array}$ & Suicide vector & $\begin{array}{l}\mathrm{Km}^{r} \text { mWasabi } \\
\text { tdTomato }\end{array}$ & $\mathrm{n} / \mathrm{a}$ & Viljoen et al. (2018) \\
\hline $\begin{array}{l}\text { M. avium (JCM34) (Leucine } \\
\text { auxotroph) }\end{array}$ & Transduction & pсаA & $\begin{array}{l}\text { Phasmid derived from } \\
\text { pHAE87 }\end{array}$ & $\mathrm{Hyg}^{r}$ leuD & $\mathrm{n} / \mathrm{a}$ & Otero et al. (2003) \\
\hline \multicolumn{7}{|l|}{ Two-step allelic exchange } \\
\hline M. avium (920A6) & Electroporation & $r t f A$ & $\begin{array}{l}\text { Conditionally replicating } \\
\text { vector (ts-oriM) }\end{array}$ & $\mathrm{Hyg}^{r} x y / E$ & $\operatorname{sac} B$ & Irani et al. (2004) \\
\hline M. avium (104) & Electroporation & $m t f D$ & $\begin{array}{l}\text { Conditionally replicating } \\
\text { vector (ts-oriM) }\end{array}$ & $\mathrm{Km}^{r}$ & $\operatorname{sac} B$ & $\begin{array}{l}\text { Krzywinska et al. } \\
(2005)\end{array}$ \\
\hline M. chelonae (ATCC 35752) & Electroporation & $\begin{array}{l}\mathrm{MCH} H_{4} 4689 \mathrm{c} \\
\mathrm{MCH} 4690 \mathrm{c} \\
\mathrm{MCH}_{-} 4691 \mathrm{c}\end{array}$ & $\begin{array}{l}\text { Conditionally replicating } \\
\text { vector (ts-oriM) }\end{array}$ & $\mathrm{Km}^{r}$ or Zeo ${ }^{r} x y l E$ & $\operatorname{sac} B$ & Moura et al. (2014) \\
\hline M. marinum & Electroporation & esxB-esxA & Suicide vector & $\mathrm{Hyg}^{r}$ lacZ & $\operatorname{sac} B$ & Zhang et al. (2016) \\
\hline $\begin{array}{l}\text { M. abscessus } \\
\text { (ATCC19977) }\end{array}$ & Electroporation & $\begin{array}{l}\text { MAB_4395 } \\
{\left[\operatorname{aac}\left(2^{\prime}\right)\right]}\end{array}$ & Suicide vector & Apr $r^{r}$ & katG & $\begin{array}{l}\text { Rominski et al. } \\
(2017)\end{array}$ \\
\hline $\begin{array}{l}\text { M. abscessus } \\
\text { (ATCC19977) }\end{array}$ & Electroporation & $\begin{array}{l}\text { mmpL4b mbtH } \\
\text { MAB_2875 } \\
\text { MAB_2833 }\end{array}$ & Suicide vector & $\mathrm{Km}^{r} \mathrm{lac} Z$ & galK & $\begin{array}{l}\text { Gregoire et al. } \\
(2017)\end{array}$ \\
\hline
\end{tabular}


several months are required to generate mutants in slow-growing mycobacteria (defined as those that take $>7$ days to form colonies on a plate).

\section{RECOMBINEERING}

Recombineering is a homologous recombination process mediated by the expression bacteriophage-encoded recombination enzymes in bacterial cells. In this method, the AESs are linear DNA fragments, which are either doublestranded (dsDNA) or single-stranded (ssDNA), depending on the phage proteins being used (Murphy, 1998). Two recombineering systems are commonly used in E. coli namely, the lamda $(\lambda)$ Red system, comprised of three proteins, Exo, Beta and Gam (Yu et al., 2000), and the Rac prophage system, which requires RecE and RecT proteins (Zhang et al., 1998). The Exo and RecE exonucleases function to generate a ssDNA tail or overhangs from a linear dsDNA, while the RecT and Beta proteins bind to ssDNA promoting annealing to the homologous chromosomal region (Figure 1). The $\lambda$ Red Gam protein inhibits the host's recombination system to prevent dsDNA degradation. Expression of phage-encoded recombineering proteins in $E$. coli increases recombination efficiency between 10- and 100-fold (Zhang et al., 1998; Yu et al., 2000).

The $\lambda$ Red and Rac systems do not function efficiently in mycobacteria, and therefore homologs from the mycobacteriophage Che9c, gp60 (RecE homolog) and gp61 (RecT homolog), were investigated for their ability to mediate recombineering (van Kessel and Hatfull, 2007; van Kessel et al., 2008). Recombineering with a dsDNA substrate in M. smegmatis revealed that while efficiency was dependent on the length of homology (increased efficiency with AESs between 50 bp and $500 \mathrm{bp}$ ), the absolute number of transformants recovered was limited by the competence of the cells. An AES substrate containing 500 bp of homology on either side of a hygromycin gene yielded recombination frequencies of between $2.4 \times 10^{-5}$ and $2.8 \times 10^{-4}$, with $>90 \%$ of the colonies being recombinants (van Kessel and Hatfull, 2007). Similar frequencies were observed in the slow-grower, M. tuberculosis. The inclusion of res, FRT or loxP sites on either sides of the antibiotic resistance gene in the dsDNA AES facilitates removal of the marker after mutant selection (van Kessel and Hatfull, 2007; Kenan, 2015). Another improvement to the system was the use of plasmids with ts-oriMs and counter-selection markers ( $s a c B)$ to facilitate curing of the gp60/gp61-expressing plasmid after recombineering, thereby preventing subsequent chromosomal rearrangements (van Kessel et al., 2008). Recombineering using short ssDNA (50-100 bp) is considerably more efficient than dsDNA recombineering (10- to 100 -fold), although this is also limited by cell competence. Single stranded AESs that target the lagging strand are more efficient, with the degree of this bias depending on chromosomal location; a 10,000-fold difference has been observed in some loci (van Kessel et al., 2008). Unlike dsDNA recombineering, ssDNA recombineering in slow growing mycobacteria is 5 - to 10 -fold less efficient than
M. smegmatis (van Kessel and Hatfull, 2008). Since these short AESs do not carry a selection marker, identification of mutants can require screening of up to a 1000 single clones in the absence of a selectable phenotype, which is a major drawback of this technique (Kenan, 2015).

Currently, very few reports of recombineering in NTMs exist in the literature. Double stranded recombineering was successfully used in $M$. chelonae (ATCC 35752) to disrupt three porin genes (MCH_4689c, MCH_4690c, and $\left.M C H \_4691 c\right)$ using a plasmid expressing gp60 and gp61 (Moura et al., 2014). Electroporation of the ATCC 35752 strain with $300 \mathrm{ng}$ of each AES yielded between 29 and $65 \mathrm{Zeo}^{\mathrm{r}}$ colonies, and between 30 and $80 \%$ of these colonies had undergone allelic exchange. Interestingly, the plasmid expressing the recombineering proteins was rapidly lost from $M$. chelonae in the absence of selection (1-2 passages), and therefore counter-selection or the use of conditionally replicating plasmids was not required. Use of dsDNA recombineering to disrupt $m m p L 4 b$ in $M$. abscessus (CIP104536T) was significantly less efficient, with only $7 \%$ of clones undergoing allelic exchange. The reason for the low efficiency in $M$. abscessus is unclear and suggests that the utility of the method may be species-specific. To date, the only report of ssDNA recombineering in NTMs is in M. marinum, where it was used in combination with CRISPR/Cas12a, and is discussed below.

\section{OLIGONUCLEOTIDE-MEDIATED RECOMBINEERING FOLLOWED BY BXb1 INTEGRASE TARGETING (ORBIT)}

Oligonucleotide-mediated recombineering followed by Bxb1 integrase targeting (ORBIT) was developed by combining of two efficient recombination systems, namely homologous recombination and site-specific integration (Murphy et al., 2018). The technique is mediated by the co-expression of the gp61 annealase and site-specific Bxb1 integrase, and involves co-transformation of a ssDNA targeting oligonucleotide (containing a Bxb1 recombination site) and a payload plasmid (containing an antibiotic selectable marker and a Bxb1 recombination site) (Figure 1). The gp61 annealase mediates recombineering of the oligonucleotide to introduce a Bxb1 site in a site-specific manner, while Bxb1 facilitates concomitant integration of the payload plasmid (Murphy et al., 2018). A library of payload plasmids was generated to facilitate use of the technique for promoter replacements, deletions and the introduction of C-terminal fusions. Application of ORBIT in M. smegmatis and M. tuberculosis yielded 20-200 clones for each transformation (using $1 \mu \mathrm{g}$ oligonucleotide and $200 \mathrm{ng}$ payload plasmid). In ORBIT, the Bxbl integration system is independent of host factors because both the integrase and recombination sites are provided. The feasibility of using ORBIT in NTM species would therefore depend on the efficiency of the gp61 to mediate recombineering. 

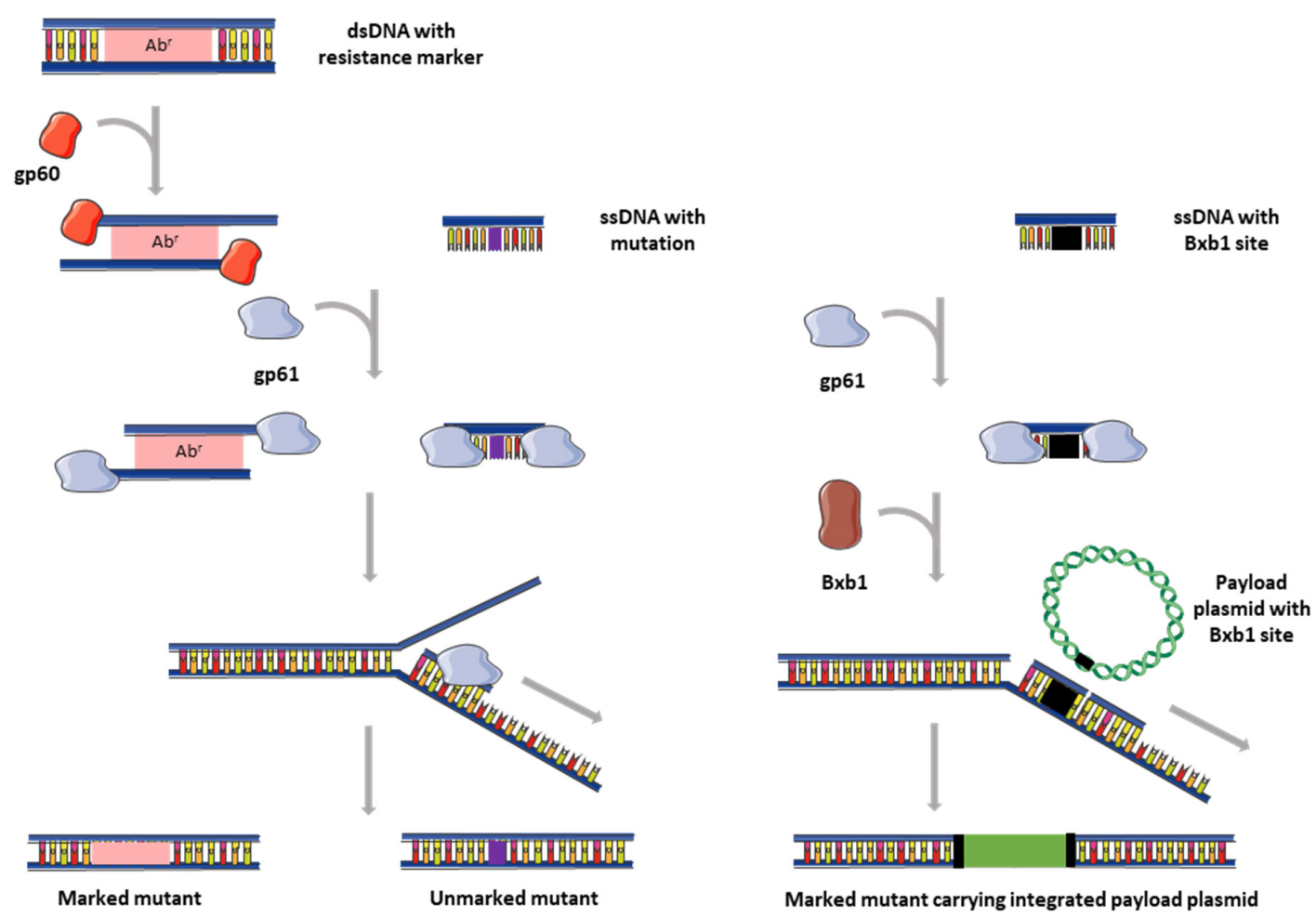

Marked mutant carrying integrated payload plasmid

FIGURE 1 | Graphical representation of recombineering with ds and SSDNA and ORBIT. dsDNA substrates (left) usually contain an antibiotic resistance marker for selection, while ssDNA substrates (middle) carry only the desired mutation flanked by short regions (25-50 bp) of homology. In ORBIT (right), the ssDNA substrate contains a Bxb1 integration site flanked by short regions (25-50 bp) of homology. The gp60 exonuclease (RecE homolog) generates single stranded overhangs from the dsDNA fragments, while gp61 (RecT homolog) promotes annealing of ssDNA to homologous regions in the chromosome. Recombineering is more efficient when oligonucleotides are targeted to the lagging strand (indicated in diagram). In ORBIT, Bxb1 facilitates concomitant integration of the payload plasmid. Images adapted from Servier Medical Art by Servier which is licensed under a Creative Commons Attribution 3.0 Unported License (https://smart.servier.com/).

\section{RANDOM MUTAGENESIS USING TRANSPOSONS}

Transposons are mobile genetic elements that in addition to the genes essential for transposition, also have genes encoding a phenotypic trait, such as antibiotic resistance. Two types of transposition events exist, namely replicative and cut-and-paste (conservative) transposition (Griffiths et al., 2000). In replicative transposition co-integration between the transposon delivery replicon and the target DNA occurs in such a way that transposon is duplicated. A site-specific recombinase subsequently resolves the co-integrant to reform the delivery replicon, leaving a single copy of the transposon in the target DNA. In cut-and-paste transposition, a transposase binds to a transposon creating a synaptic complex, cleaving it from the donor molecule and inserting it into the target site without duplication. Cut-and-paste transposition is typical for $\mathrm{Tn} 5, \mathrm{Tn} 10$ and mariner transposons.

The first insertional mutant libraries in $M$. smegmatis $\left(m c^{2} 155\right)$ were created using the Tn611 transposon, a derivative of the Mycolicibacterium fortuitum transposon Tn610 (Martin et al., 1990). Initially the transposon was used with nonreplicating plasmids for mutagenesis, but this resulted in low transposition efficiency. Combining Tn611 with a conditionally replicating plasmid (ts-oriM) improved efficiency by facilitating initial selection of transformants at $30^{\circ} \mathrm{C}$ and subsequent selection of mutants at $39^{\circ} \mathrm{C}$ (non-permissive temperature). Transposition of Tn611 occurs by the replicative mechanism, and in M. smegmatis, no insertion hotspots were observed (Guilhot et al., 1994). Culturing of transposon mutants without antibiotic selection revealed a low reversion frequency, indicating that Tn611 stabilizes upon host chromosome integration (Guilhot et al., 1994). The transposons Tn5367, Tn5368 and Tn5370 are derived from the M. smegmatis IS1096 insertion sequence and differ from Tn611 in that the mechanism of transposition is cut-and-paste (McAdam et al., 1995). Transposon insertion is random, and analysis of the insertion sites identified a weak consensus sequence for insertion ( $\left.5^{\prime}-\mathrm{NNP} y(\mathrm{~A} / \mathrm{T}) \mathrm{A}(\mathrm{A} / \mathrm{T}) \mathrm{NN}-3^{\prime}\right)$, showing a preference for an AT-rich center.

As for allelic exchange, temperature sensitive phages, such as phAE77 and phAE94, have been used for more efficient transposon delivery. In M. marinum, transduction with phAE94 carrying Tn5367 yielded $10^{5} \mathrm{Km}^{\mathrm{r}}$ colonies per $\mathrm{ml}$ of the transduction mixture. This was significantly more efficient than the phAE77 derivative which yielded only $10^{3}$ per $\mathrm{ml}$ (Rybniker et al., 2003), demonstrating that phage choice influences efficiency (Table 2). Transposition efficiency is also influenced by experimental conditions. For example, in M. avium subsp. paratuberculosis (K-10) strain optimal transposition 
TABLE 2 | Examples of random transposon mutagenesis in non-tuberculosis mycobacteria (NTMs).

\begin{tabular}{|c|c|c|c|c|c|}
\hline Mycobacterial species & Transposon & Plasmid/phage & $\begin{array}{l}\text { Selection } \\
\text { marker(s) }\end{array}$ & Purpose/selection & References \\
\hline M. marinum & IS1096 IS6110 & $\begin{array}{l}\text { pYUB285 pUS252 (suicide } \\
\text { plasmids) }\end{array}$ & $\mathrm{Km}^{\mathrm{r}}$ & Insertional library & $\begin{array}{l}\text { Talaat and Trucksis } \\
(2000)\end{array}$ \\
\hline M. marinum & $\operatorname{Tn} 5367$ & $\begin{array}{l}\text { phAE94 (TM4) phAE77 (D29) } \\
\text { (ts phasmids) }\end{array}$ & $\mathrm{Km}^{\mathrm{r}}$ & Insertional library & Rybniker et al. (2003) \\
\hline M. marinum & Mos1 & pM272B (ts-oriM) & $\mathrm{Km}^{\mathrm{r}} \mathrm{sacB}$ & $\begin{array}{l}\text { Insertional library, pigmentation } \\
\text { mutants }\end{array}$ & Gao et al. (2003) \\
\hline M. marinum & MycoMarT7 & phAE94 (TM4) & $\mathrm{Km}^{r}$ & Essentiality screen & $\begin{array}{l}\text { Weerdenburg et al. } \\
\text { (2015) }\end{array}$ \\
\hline $\begin{array}{l}\text { M. avium subsp. } \\
\text { paratuberculosis }\end{array}$ & $\operatorname{Tn} 5367$ & phAE94 (TM4) & $\mathrm{Km}^{\mathrm{r}}$ & Insertional library & Harris et al. (1999) \\
\hline $\begin{array}{l}\text { M. avium subsp. } \\
\text { paratuberculosis }\end{array}$ & Tn5367 MycoMarT7 & phAE94 (TM4) phAE94 (TM4) & $\mathrm{Km}^{r}$ & Insertional library & Rathnaiah et al. (2016) \\
\hline M. avium & MycoMarT7 & phAE94 (TM4) & $\mathrm{Km}^{\mathrm{r}}$ & $\begin{array}{l}\text { Insertional library, antibiotic } \\
\text { susceptibility }\end{array}$ & Cangelosi et al. (2006) \\
\hline $\begin{array}{l}\text { M. avium subsp. } \\
\text { hominissuis }\end{array}$ & MycoMarT7 & phAE94 (TM4) & $\mathrm{Km}^{r}$ & Essentiality screen & Dragset et al. (2019) \\
\hline $\begin{array}{l}\text { M. avium subsp. avium } \\
\text { (HMC02) }\end{array}$ & EZ::TN & $\mathrm{n} / \mathrm{a}$ & $\mathrm{Km}^{\mathrm{r}}$ & Insertional library Ciprofloxacins & Cangelosi et al. (2006) \\
\hline M. kansasii & MycoMarT7 & phAE94 (TM4) & $\mathrm{Km}^{r}$ & $\begin{array}{l}\text { Insertional library, colony } \\
\text { morphology }\end{array}$ & Budell et al. (2020) \\
\hline M. intracellulare & MycoMarT7 & phAE94 (TM4) & $\mathrm{Km}^{r}$ & Essentiality screen & Tateishi et al. (2020) \\
\hline M. fortuitum & TnphoA & pRT291 (suicide vector) & $\mathrm{Km}^{\mathrm{r}}$ phoA & $\begin{array}{l}\text { Library with insertions in } \\
\text { secreted/membrane proteins, } \\
\text { resistance to acid stress biofilm } \\
\text { defect }\end{array}$ & $\begin{array}{l}\text { Poonam et al. (2019); } \\
\text { Katoch et al. (2020) }\end{array}$ \\
\hline M. ulcerans & $\operatorname{Tn} 5367$ & phAE94 (TM4) & $\mathrm{Km}^{\mathrm{r}}$ & Unsuccessful & Rybniker et al. (2003) \\
\hline
\end{tabular}

frequencies $\left(10^{-6}\right.$ to $10^{-7}$ per recipient cell) were obtained after $4 \mathrm{~h}$ of co-incubation of bacteria and phages, while the frequency dropped 10-fold with longer incubation times (Harris et al., 1999). Tn5367 mutagenesis in both M. marinum and $M$. avium subsp. paratuberculosis $(K-10)$ revealed that insertions were flanked with $8 \mathrm{bp}$ target duplication, and in M. marinum insertion of small parts of the phage DNA was observed in some mutants (Harris et al., 1999; Rybniker et al., 2003). Use of the phAE94/Tn5367 system in Mycobacterium ulcerans resulted in a high number of mutants with slow growth, limiting DNA isolation for Southern blot hybridization (Rybniker et al., 2003). Although PCR screening suggested transposon insertion, plaque formation at $32^{\circ} \mathrm{C}$ led the authors to speculate that integration of the prophage had occurred. phAE94 may therefore be functioning as a temperate phage in $M$. ulcerans, highlighting the need to confirm temperaturesensitive replication in different species.

Mariner transposons have recently been developed as genetic tools for use in bacteria and the most commonly used are Mos1 and Himar1. One of the major advantages of the mariner transposons are their reduced recognition sequence $\left(5^{\prime}-\mathrm{TA}-3^{\prime}\right)$, which is less restrictive. The first mariner transposon used for mutagenesis in M. marinum was Mos1 isolated from Drosophila melanogaster. A delivery vector, pM272B, was engineered to contain the transposon with a kanamycin resistance gene, a thermosensitive origin of replication and a $\operatorname{sac} B$ gene used for counter selection (Gao et al., 2003). Following initial selection of kanamycin $(\mathrm{Km})$ resistant transformants after electroporation, transposition was observed at a rate of approximately $3 \times 10^{2} \mathrm{Km}$ resistant and sucrose sensitive bacteria per $10^{5} \mathrm{Km}$ resistant bacteria. Analysis of the transposon junctions revealed a TA dinucleotide insertion site followed by GC-rich genomic sequences. The mariner transposon inserted randomly, usually with one transposon copy per genome, and the delivery vector was lost upon transposition.

The most-frequently used transposon in mycobacterial research is the Himar-1-derived MycoMarT7 mariner transposon. This transposon has been engineered to contain a T7 promoter, used for insertion site identification, and a kanamycin selection marker. It is usually delivered using a temperature-sensitive phage. A comparison of Tn5367 and the MycoMarT7 mariner transposon in M. avium subsp. paratuberculosis revealed a $>3$-fold higher number of insertions for MycoMarT7, and a higher percentage of insertions occurring within genes ( $83 \%$ vs. $74 \%)$ (Rathnaiah et al., 2016). The MycoMarT7 transposon is therefore superior at creating a comprehensive library. Despite the reduced recognition sequence, transposition bias was detected for MycoMarT7, and it was estimated that 400,000 M. avium subsp. paratuberculosis mutants would be required for a representative library. This is four times higher than the estimate for M. tuberculosis and such biases could result in an overestimation of the number of essential genes in forward genetic screens in this organism. Evaluation of $\Phi$ MycoMarT7 in Mycobacterium kansasii revealed 
that while temperature sensitivity was maintained, plaque formation in M. kansasii was 800-fold less efficient than for M. smegmatis (Budell et al., 2020). Mapping of the insertion sites for the $14,700 \mathrm{M}$. kansasii mutants revealed that $82 \%$ of the insertions in the chromosome were in open reading frames (ORFs); a similar percentage (83\%) was observed for the pMK12478 plasmid present in this strain. The slightly higher percentage of insertions in annotated genes in the plasmid (74\% vs. $62 \%$ ) could be due to the non-essential nature of genes present extra-chromosomally, however, since the library is not saturated the data is not conclusive.

Most studies in NTMs have used transposon mutagenesis to select for mutants with specific phenotypes. Examples include pigmentation variants in $M$. marinum (Gao et al., 2003), M. kansasii mutants with altered colony morphology (Budell et al., 2020) and M. avium mutants with altered antibiotic susceptibility (Cangelosi et al., 2006) (see Table 2 for details). The TnphoA transposon was developed to perform more targeted screens that identify mutants with insertions in membrane or secreted proteins (Taylor et al., 1987). The TnphoA transposon encodes an alkaline phosphatase enzyme, but the gene is lacking its signal sequence (Katoch et al., 2020). If the transposon inserts into a gene encoding a membrane or secreted protein, the presence of alkaline phosphatase at the cell surface turns the colony blue when selected on 5-bromo-4chloro-3-indolyl phosphate (XP). A TnphoA transposon mutant library in $M$. fortuitum yielded 42 mutants with alkaline phosphatase activity, approximately $9 \%$ of the total number of mutants isolated (Katoch et al., 2020). Analysis of a mutant (MT721) with a biofilm formation defect identified a deletion in the membrane protein anthranilate phosphoribosyl transferase (TrpD), confirming the utility of this approach (Katoch et al., 2020).

One of the limitations of using transposons for mutagenesis is that a functional transposase must be produced within the host. The use of transposomes, which is a stable complex between a purified transposase enzyme and the transposon, overcomes this limitation (Goryshin et al., 2000). A commercial transposome system derived from Tn903, EZ-Tn (Epicenter), has been developed for use in a broad host range, and was used to generate a mutant library of 3500 mutants in the M. avium subsp. avium HMC02 (Laurent et al., 2003). The efficiency of transposition efficiency of EZ-Tn in certain $M$. avium morphotypes was significantly lower, presumably due to transposome delivery being influenced by cell wall composition. Low transformation efficiency is therefore a limitation of this system.

In addition to generating mutants, transposon mutagenesis can be used for the genome-wide prediction of essential genes. This involves the generation of saturating transposon mutant libraries (hundreds of thousands of mutants), and the mapping of the transposon insertion site in every mutant in the library. By comparing the frequency of observed insertions to all possible insertion sites within a gene, essential genes can be identified. In addition, genes that confer a growth advantage or defect can be identified by comparing the abundance of mutants in the library. This approach has been used with the MycoMarT7 transposon to identify essential genes in M. marinum (Weerdenburg et al.,
2015), Mycobacterium avium subsp. hominissuis (Dragset et al., 2019) and Mycobacterium intracellulare (Tateishi et al., 2020) (see Table 2 for details).

\section{RANDOM MUTAGENESIS BY NON-HOMOLOGOUS RECOMBINATION}

Although high levels non-homologous recombination is undesirable when introducing site-specific mutations, its nonspecific nature can be harnessed to introduce random mutations. The high levels of non-homologous recombination in slowgrowing mycobacteria has been exploited to generate mutants in M. bovis (Wilson et al., 1997) and M. avium (Khattak et al., 2012). The study in M. bovis was initially aimed at investigating the role of the $a p h C$ gene, and therefore used an AES containing a kanamycin resistance gene (neo) flanked by sequences from the $M$. bovis aphC gene. Of the 440 transformants containing an insertion, none disrupted the $a p h C$ gene, indicating that non-homologous rather than homologous recombination had occurred (Wilson et al., 1997). Since the flanking regions did not appear to confer any specificity, the study in $M$. avium subsp. hominissuis used a linear DNA fragment containing a hygromycin resistance gene flanked by vector (pYUB854) sequences. Electroporation of 3-6 $\mu \mathrm{g}$ of this linear DNA fragment yielded about $1000 \mathrm{Hyg}^{\mathrm{r}}$ colonies (Khattak et al., 2012). Analysis of 13 randomly chosen mutants revealed that the resulting deletions ranged between 2 and 669 bp, and 12 of the 13 deletions had disrupted a single gene. While this method is easier to perform than transposon mutagenesis, it is significantly less efficient.

\section{MUTAGENESIS USING CRISPR/Cas}

The Clustered Regularly Interspaced Palindromic Repeats (CRISPR)/CRISPR associated protein (Cas) systems in prokaryotes act as an adaptive immune system which renders bacteria resistant to repeated phage infection (Marraffini, 2015). Following infection, phage DNA is digested by the host and selected fragments are integrated into the CRISPR locus as short sequences, termed spacers (Bolotin et al., 2005; Mojica et al., 2005; Pourcel et al., 2005). The integrated DNA is transcribed to produce a mature transcript, crRNA, which will direct the Cas nuclease to cleave sequences that are complementary to the crRNA in the phage genome during subsequent infections. The specificity and programmability of the CRISPR/Cas system makes it an attractive tool for mutagenesis, and the CRISPR/Cas9 and CRISPR/Cas12a systems have been widely utilized to introduce mutations in a range of organisms. By using synthetic small guide RNAs (sgRNA) to mimic crRNA, a Cas-sgRNA complex can be directed to a specific gene by Watson-Crick base pairing between the sgRNA and the target DNA sequence (Figure 2). The Cas nuclease then produces a double strand break (DSB) in the target gene, which must be repaired by the host to maintain chromosomal integrity. The DSB repair is 


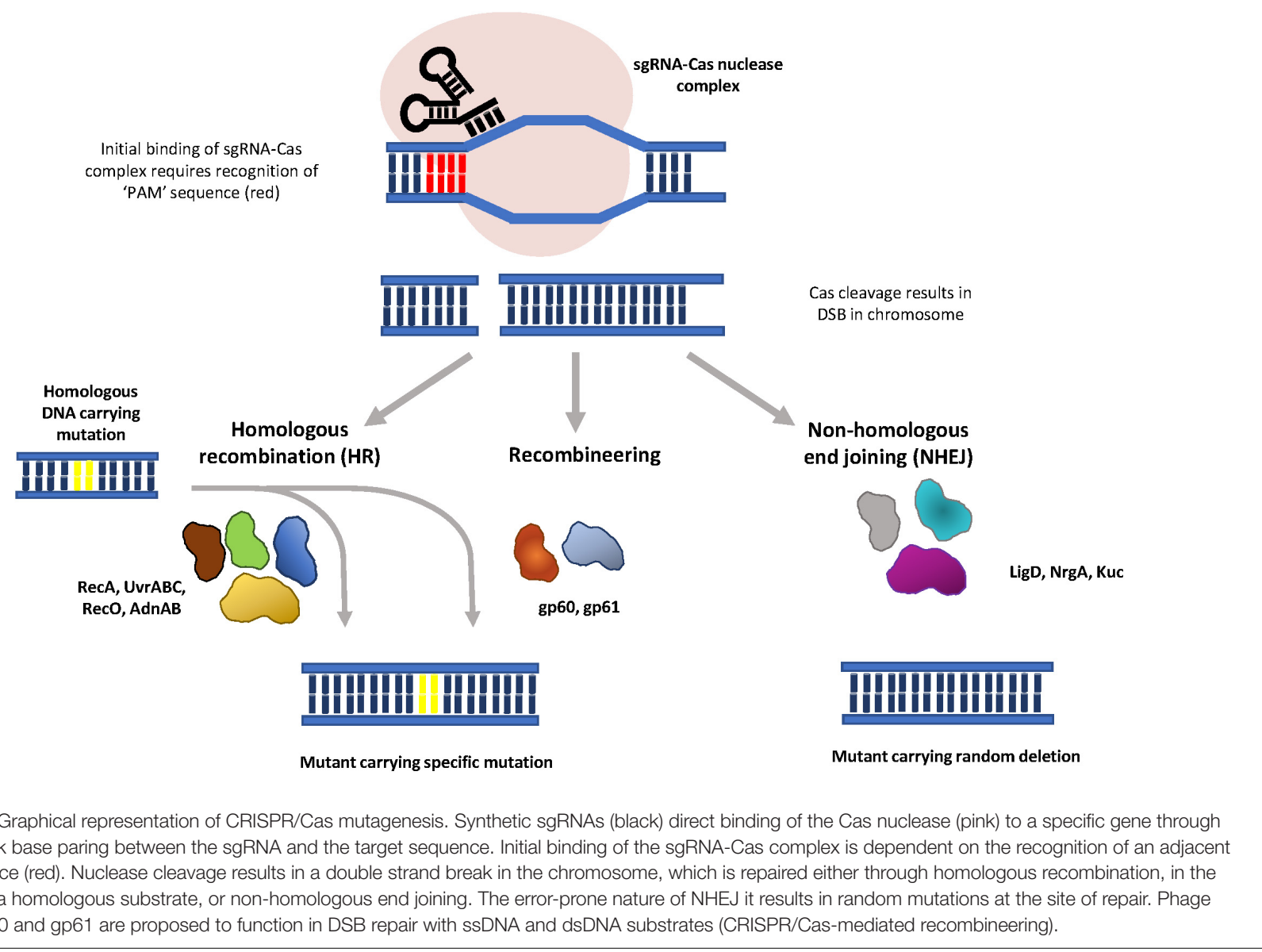

performed either by homologous recombination (HR), using a corresponding homologous template, or by non-homologous end joining (NHEJ), in absence of a homologous template (Featherstone and Jackson, 1999). Because NHEJ is prone to insertions and deletions, this can lead to mutations in the target gene.

The first reported use of the CRISPR/Cas9 system in mycobacteria was for gene silencing by CRISPR interference (CRISPRi) (Choudhary et al., 2015). Genes can be silenced by mutating the endonuclease domain of the Cas9 to make it catalytically inactive (dCas9) (Larson et al., 2013). The sgRNAdCas9 complex binds to the target DNA sequence, however, since dCas9 cannot cleave the DNA, the complex remains bound, blocking the binding or movement of RNA polymerase. This results in gene-specific transcriptional repression by blocking either initiation or elongation, depending on the position of the sgRNA-dCas9 complex relative to the start of the gene (Qi et al., 2013). Evaluation of 11 Cas9 proteins in mycobacteria revealed that the enzyme from Streptococcus thermophilus (dCas9 ${ }_{\text {Sth1 }}$ ) was most efficacious in the knockdown of endogenous gene expression (Rock et al., 2017). CRISPRi is a major advance in studying gene function due to its simplicity. The technique has one major drawback in that when genes are operonic, all genes downstream of the target gene are also silenced.
In most DNA-targeting CRISPR-Cas systems, initial binding requires recognition of a protospacer adjacent motif (PAM), a short ( 3 to $5 \mathrm{bp}$ ) DNA sequence immediately upstream of the sequence recognized by the crRNA (Marraffini, 2015). Since Cas9 and Cas12a nucleases recognize different PAMs (Leenay et al., 2016), using different Cas nucleases facilitates targeting of different sites in the chromosome. The CRISPR/Cas12a system was first used in $M$. smegmatis to increase recombineering efficiency (Yan et al., 2017). A two-plasmid system, (also demonstrated to function in E. coli and Yersinia pestis) coupled an Cas12a endonuclease from Francisella novicida (FnCpf1) with the recombineering proteins gp60 and gp61 (Figure 2), to create markerless, scarless mutations. Recombineering using ssDNA was 50-times more efficient when combined with FnCpfl-site specific cleavage. The expected strand bias was observed, with efficiencies of $80 \%$ and $69 \%$ obtained for oligonucleotides targeted to the lagging strand, while the leadingstrand oligonucleotide had a fivefold lower efficiency. The efficiency of introducing insertions and deletions by CRISPRFnCpf1-enhanced recombineering is size - dependent. For insertions, $27 \%$ of transformants had 5 bp insertions, $10 \%$ had $10 \mathrm{bp}$ insertions, while only $3.1 \%$ had $20 \mathrm{bp}$ insertions (Yan et al., 2017). Similarly, the efficiency for introducing small deletions $(5,10,20 \mathrm{bp})$ ranged between $70 \%$ and $90 \%$, while significantly lower efficiencies of $17.4 \%$ and $8.2 \%$ were 
observed for $418 \mathrm{bp}$ and $1000 \mathrm{bp}$ deletions, respectively. In addition, CRISPR-FnCpf1-enhanced recombineering using $1 \mathrm{~kb}$ dsDNA fragments facilitated the introduction of markerless deletions of up to $4000 \mathrm{~kb}$ with an efficiency of greater than 45\% (Yan et al., 2017). The limitation of the Yan et al. (2020) study was that the requirement for gp60 and gp61 was not demonstrated, and therefore the role of endogenous homologous recombination proteins in the process is not clear. The major advantage of CRISPR/Casenhanced recombineering is that it takes approximately half the time of two-step allelic exchange, however, since it did not work in $M$. tuberculosis, it may not function in all NTMs (Yan et al., 2020).

The FnCpf1 nuclease was unable to generate mutants in M. smegmatis in the absence of recombineering substrates, suggesting that NHEJ was not functioning to repair DSB generated by the nuclease (Sun et al., 2018). In contrast, expression of the FnCpf1 nuclease and a crRNA targeting a non-essential gene in $M$. marinum resulted in deletions ranging from 2 bp to 10179 bp (Yan et al., 2020). Deletion of $k u$ and $\operatorname{lig} D$ genes in $M$. marinum reduced genome editing efficiency to below $10 \%$, while complementation increased NHEJ editing efficiency to 90\%. Furthermore, expression of the M. marinum NHEJ (MmNHEJ) locus with the CRISPRFnCpf1 system in M. smegmatis resulted mutations in $0.75 \%$ of transformants. This efficiency was increased to $90 \%$ when the experiment was performed in a recA null mutant, suggesting that inhibiting $\mathrm{HR}$ is required for the NHEJ system to repair DSBs in M. smegmatis.

To facilitate efficient CRISPR-FnCpf1-assisted NHEJ genome editing in mycobacteria a two-plasmid system was subsequently developed; one plasmid expresses $\operatorname{Rec}_{\mathrm{mu}}$ (dominant RecA negative mutant), RecX (RecA regulator), $M m$ NHEJ ( $M$. marinum NHEJ locus), while the other expresses FnCpf1 and the crRNA (Yan et al., 2020). These promoted the NHEJ genome editing in $M$. smegmatis with high efficiency (80-90\%). Replacement of the FnCpf1 with Cas9 9 Sth1 (Cas9 from Streptococcus thermophilus) achieved genome editing efficiencies of above $80 \%$ in both M. smegmatis and M. tuberculosis, and deletions of between 1 and $324 \mathrm{bp}$ observed at the cleavage site in $M$. tuberculosis. The method may therefore have some utility for introducing random mutations in other mycobacterial species.

\section{CONCLUDING REMARKS}

Despite advances in mycobacterial mutagenesis, a limiting factor for all techniques is the efficient delivery of DNA into cells. Due to the complex nature of the mycobacterial cell wall, electroporation efficiency is low in many species. The M. smegmatis $\mathrm{mc}^{2} 155$ strain, which has become a workhorse in mycobacterial research, is a mutant that was isolated for its high transformation efficiency, an efficiency $10^{5}$ times greater than its parental strain (Snapper et al., 1990). Transformation efficiency differs both between mycobacterial species and between strains within a species. For example, strains of $M$. avium display transformation efficiencies between $10^{2}$ and $10^{4}$ (Foley-Thomas et al., 1995; Lee et al., 2002), while efficiencies of $10^{5}$ have been reported for $M$. bovis BCG and M. tuberculosis H37Rv (Wards and Collins, 1996). Although optimization of the electroporation procedure may afford some improvement (Wards and Collins, 1996; Lee et al., 2002), in some species the isolation of transformation efficient mutants may be required (Snapper et al., 1990; Mahenthiralingam et al., 1998; Dragset et al., 2019). Delivery of DNA using conditionally replicating mycobacteriophages is a more efficient alternative to electroporation, although this requires additional steps to propagate the phages in a suitable host. Most of the phasmids used in mycobacterial research are derived from phage TM4, which has a broad host range. Differences in transfection efficiencies between species and strains have been observed (Foley-Thomas et al., 1995; Dragset et al., 2019) and optimization of transduction protocols may be required (Park et al., 2008). Another consideration when using temperature sensitive phages, is the functionality of the ts-oriM in the mycobacterial species being manipulated (Rybniker et al., 2003).

Differences in the physiology of mycobacterial species can impact on the utility of a mutagenesis technique and should therefore be considered when choosing an approach. Examples here include the functionality of selection markers (such as $s a c B$ in $M$. abscessus) or the rate of spontaneous antibiotic resistant mutants arising in an organism; a property determined both by the mechanisms of resistance and the DNA repair pathways in that species. The differential functionality of CRISPR-mediated mutagenesis in M. smegmatis, M. tuberculosis and $M$. marinum highlights how differences in DNA repair mechanisms in different species can influence the choice of technique (Yan et al., 2020). In M. smegmatis, CRISPR-FnCpf1 cleavage could enhance recombineering, however, the system did not work in M. tuberculosis. Similarly, the NHEJ pathway could repair DSB introduced by the FnCpf1 nuclease in $M$. marinum, but not in $M$. smegmatis. Given that studies investigating the physiology of NTMs remain limited, choice of mutagenesis techniques in many species often involves trial and error. A concerted effort to both optimize mutagenesis tools for use in NTMs and to understand the underlying physiology influencing their efficacy, is therefore needed to advance the genetic manipulation of NTMs.

\section{AUTHOR CONTRIBUTIONS}

NC wrote manuscript. MW wrote and edited manuscript. Both authors contributed to the article and approved the submitted version.

\section{FUNDING}

This work was supported by a Building Research Active Academic Staff (B.R.A.A.S.) Grant awarded to MW from the University of Cape Town. 


\section{REFERENCES}

Alonso, M. N., Malaga, W., Mc Neil, M., Jackson, M., Romano, M. I., Guilhot, C., et al. (2020). Efficient method for targeted gene disruption by homologous recombination in Mycobacterium avium subspecie paratuberculosis. Res. Microbiol. 171, 203-210. doi: 10.1016/j.resmic.2020. 04.001

Bardarov, S., Bardarov, S., Pavelka, M. S., Sambandamurthy, V., Larsen, M., Tufariello, J., et al. (2002). Specialized transduction: an efficient method for generating marked and unmarked targeted gene disruptions in Mycobacterium tuberculosis, M. bovis BCG and M. smegmatis. Microbiology 148, 3007-3017. doi: 10.1099/00221287-148-10-3007

Bolotin, A., Quinquis, B., Sorokin, A., and Ehrlich, S. D. (2005). Clustered regularly interspaced short palindrome repeats (CRISPRs) have spacers of extrachromosomal origin. Microbiol. Read. Engl. 151, 2551-2561. doi: 10.1099/ mic. $0.28048-0$

Budell, W. C., Germain, G. A., Janisch, N., McKie-Krisberg, Z., Jayaprakash, A. D., Resnick, A. E., et al. (2020). Transposon mutagenesis in Mycobacterium kansasii links a small RNA gene to colony morphology and biofilm formation and identifies 9,885 intragenic insertions that do not compromise colony outgrowth. MicrobiologyOpen 9:e988. doi: 10.1002/ mbo3.988

Cangelosi, G. A., Do, J. S., Freeman, R., Bennett, J. G., Semret, M., and Behr, M. A. (2006). The two-component regulatory system mtrAB is required for morphotypic multidrug resistance in Mycobacterium avium. Antimicrob. Agents Chemother. 50:461. doi: 10.1128/AAC.50.2.461-468.2006

Choudhary, E., Thakur, P., Pareek, M., and Agarwal, N. (2015). Gene silencing by CRISPR interference in mycobacteria. Nat. Commun. 6:6267. doi: 10.1038/ ncomms 7267

Dragset, M. S., Ioerger, T. R., Loevenich, M., Haug, M., Sivakumar, N., Marstad, A., et al. (2019). Global Assessment of Mycobacterium avium subsp. hominissuis genetic requirement for growth and virulence. mSystems 4, 1-16. doi: 10.1128/ mSystems.00402-19

Featherstone, C., and Jackson, S. P. (1999). DNA double-strand break repair. Curr. Biol. 9, R759-R761. doi: 10.1016/S0960-9822(00)80005-6

Fedrizzi, T., Meehan, C. J., Grottola, A., Giacobazzi, E., Fregni Serpini, G., Tagliazucchi, S., et al. (2017). Genomic characterization of Nontuberculous Mycobacteria. Sci. Rep. 7:45258. doi: 10.1038/srep45258

Fine, P. E. M. (1995). Variation in protection by BCG: implications of and for heterologous immunity. Lancet 346, 1339-1345. doi: 10.5555/uri:pii: S0140673695923489

Foley-Thomas, E. M., Whipple, D. L., Bermudez, L. E., and Barletta, R. G. (1995). Phage infection, transfection and transformation of Mycobacterium avium complex and Mycobacterium paratuberculosis. Microbiology 141, 1173-1181. doi: 10.1099/13500872-141-5-1173

Gao, L.-Y., Groger, R., Cox, J. S., Beverley, S. M., Lawson, E. H., and Brown, E. J. (2003). Transposon mutagenesis of Mycobacterium marinum identifies a locus linking pigmentation and intracellular survival. Infect. Immun. 71, 922-929. doi: 10.1128/IAI.71.2.922-929.2003

Goldstone, R. M., Moreland, N. J., Bashiri, G., Baker, E. N., and Shaun Lott, J. (2008). A new Gateway ${ }^{\circledR}$ vector and expression protocol for fast and efficient recombinant protein expression in Mycobacterium smegmatis. Protein Expr. Purif. 57, 81-87. doi: 10.1016/j.pep.2007.08.015

Goryshin, I. Y., Jendrisak, J., Hoffman, L. M., Meis, R., and Reznikoff, W. S. (2000). Insertional transposon mutagenesis by electroporation of released Tn5 transposition complexes. Nat. Biotechnol. 18, 97-100. doi: 10.1038/ 72017

Gregoire, S. A., Byam, J., and Pavelka, M. S. (2017). galK-based suicide vector mediated allelic exchange in Mycobacterium abscessus. Microbiology 163, 13991408. doi: $10.1099 /$ mic. 0.000528

Griffith, D. E., Aksamit, T., Brown-Elliott, B. A., Catanzaro, A., Daley, C., Gordin, F., et al. (2007). An official ATS/IDSA statement: diagnosis, treatment, and prevention of nontuberculous mycobacterial diseases. Am. J. Respir. Crit. Care Med. 175, 367-416. doi: 10.1164/rccm.200604-571ST

Griffiths, A. J., Miller, J. H., Suzuki, D. T., Lewontin, R. C., and Gelbart, W. M. (2000). Mechanism of Transposition in Prokaryotes. Introd. Genet. Anal, 7th Edn. Available online at: https://www.ncbi.nlm.nih.gov/books/NBK21869/ (accessed October 23, 2020).
Guilhot, C., Otal, I., Van Rompaey, I., Martìn, C., and Gicquel, B. (1994). Efficient transposition in mycobacteria: construction of Mycobacterium smegmatis insertional mutant libraries. J. Bacteriol. 176, 535-539. doi: 10.1128/jb.176.2. 535-539.1994

Gupta, R. S., Lo, B., and Son, J. (2018). Phylogenomics and comparative genomic studies robustly support division of the genus Mycobacterium into an emended genus mycobacterium and four novel genera. Front. Microbiol. 9:67. doi: 10. 3389/fmicb.2018.00067

Harris, N. B., Feng, Z., Liu, X., Cirillo, S. L. G., Cirillo, J. D., and Barletta, R. G. (1999). Development of a transposon mutagenesis system for Mycobacterium avium subsp. paratuberculosis. FEMS Microbiol. Lett. 175, 21-26. doi: 10.1016 S0378-1097(99)00170-6

Hrouda, D., Baban, B., Dunsmuir, W. D., Kirby, R. S., and Dalgleish, A. G. (1998). Immunotherapy of advanced prostate cancer: a phase I/II trial using Mycobacterium vaccae (SRL172). Br. J. Urol. 82, 568-573. doi: 10.1046/j.1464410x.1998.00803.x

Irani, V. R., Lee, S.-H., Eckstein, T. M., Inamine, J. M., Belisle, J. T., and Maslow, J. N. (2004). Utilization of a ts-sacB selection system for the generation of a Mycobacterium avium serovar-8 specific glycopeptidolipid allelic exchange mutant. Ann. Clin. Microbiol. Antimicrob. 3:18. doi: 10.1186/1476-07 11-3-18

Katoch, P., Gupta, K., Yennamalli, R. M., Vashistt, J., Bisht, G. S., and Shrivastava, R. (2020). Random insertion transposon mutagenesis of Mycobacterium fortuitum identified mutant defective in biofilm formation. Biochem. Biophys. Res. Commun. 521, 991-996. doi: 10.1016/j.bbrc.2019.11.021

Kenan, M. (2015). Mycobacterial Recombineering | Springer Nature Experiments. Available online at: https://experiments.springernature.com/articles/10.1007/ 978-1-4939-2450-9_10 (accessed April 15, 2020).

Khattak, F. A., Kumar, A., Kamal, E., Kunisch, R., and Lewin, A. (2012). Illegitimate recombination: an efficient method for random mutagenesis in Mycobacterium avium subsp. hominissuis. BMC Microbiol. 12:204. doi: 10.1186/1471-2180-12204

Krzywinska, E., Bhatnagar, S., Sweet, L., Chatterjee, D., and Schorey, J. S. (2005). Mycobacterium avium 104 deleted of the methyltransferase D gene by allelic replacement lacks serotype-specific glycopeptidolipids and shows attenuated virulence in mice. Mol. Microbiol. 56, 1262-1273. doi: 10.1111/j.1365-2958. 2005.04608.x

Larson, M. H., Gilbert, L. A., Wang, X., Lim, W. A., Weissman, J. S., and Qi, L. S. (2013). CRISPR interference (CRISPRi) for sequence-specific control of gene expression. Nat. Protoc. 8, 2180-2196. doi: 10.1038/nprot.2013.132

Laurent, J.-P., Hauge, K., Burnside, K., and Cangelosi, G. (2003). Mutational analysis of cell wall biosynthesis in Mycobacterium avium. J. Bacteriol. 185, 5003-5006. doi: 10.1128/JB.185.16.5003-5006.2003

Lee, S.-H., Cheung, M., Irani, V., Carroll, J. D., Inamine, J. M., Howe, W. R., et al. (2002). Optimization of electroporation conditions for Mycobacterium avium. Tuberc. Edinb. Scotl. 82, 167-174. doi: 10.1054/tube.2002.0335

Leenay, R. T., Maksimchuk, K. R., Slotkowski, R. A., Agrawal, R. N., Gomaa, A. A., Briner, A. E., et al. (2016). Identifying and visualizing functional PAM diversity across CRISPR-Cas systems. Mol. Cell 62, 137-147. doi: 10.1016/j.molcel.2016. 02.031

Mahenthiralingam, E., Marklund, B.-I., Brooks, L. A., Smith, D. A., Bancroft, G. J., and Stokes, R. W. (1998). Site-directed mutagenesis of the 19-kilodalton lipoprotein antigen reveals no essential role for the protein in the growth and virulence of Mycobacterium intracellulare. Infect. Immun. 66, 3626-3634. doi: 10.1128/iai.66.8.3626-3634.1998

Marklund, B. I., Speert, D. P., and Stokes, R. W. (1995). Gene replacement through homologous recombination in Mycobacterium intracellulare. J. Bacteriol. 177, 6100-6105. doi: 10.1128/jb.177.21.6100-6105.1995

Marraffini, L. A. (2015). CRISPR-Cas immunity in prokaryotes. Nature 526, 55-61. doi: $10.1038 /$ nature 15386

Martin, C., Timm, J., Rauzier, J., Gomez-Lus, R., Davies, J., and Gicquel, B. (1990). Transposition of an antibiotic resistance element in mycobacteria. Nature 345, 739-743. doi: 10.1038/345739a0

Martinez, J. L., and Baquero, F. (2000). Mutation Frequencies and Antibiotic Resistance. Antimicrob. Agents Chemother. 44, 1771-1777. doi: 10.1128/AAC. 44.7.1771-1777.2000

McAdam, R. A., Weisbrod, T. R., Martin, J., Scuderi, J. D., Brown, A. M., Cirillo, J. D., et al. (1995). In vivo growth characteristics of leucine and methionine 
auxotrophic mutants of Mycobacterium bovis BCG generated by transposon mutagenesis. Infect. Immun. 63, 1004-1012. doi: 10.1128/iai.63.3.1004-1012. 1995

McFadden, J. (1996). Recombination in mycobacteria. Mol. Microbiol. 21, 205-211. doi: 10.1046/j.1365-2958.1996.6271345.x

Medjahed, H., and Reyrat, J.-M. (2009). Construction of Mycobacterium abscessus Defined glycopeptidolipid mutants: comparison of genetic tools. Appl. Environ. Microbiol. 75, 1331-1338. doi: 10.1128/AEM.01914-08

Mojica, F. J. M., Díez-Villaseñor, C., García-Martínez, J., and Soria, E. (2005). Intervening sequences of regularly spaced prokaryotic repeats derive from foreign genetic elements. J. Mol. Evol. 60, 174-182. doi: 10.1007/s00239-0040046-3

Moura, V. C. N., de Gibbs, S., and Jackson, M. (2014). Gene Replacement in Mycobacterium chelonae: application to the construction of porin knock-out mutants. PLoS One 9:e94951. doi: 10.1371/journal.pone.0094951

Murphy, K. C. (1998). Use of bacteriophage $\lambda$ recombination functions to promote gene replacement in Escherichia coli. J. Bacteriol. 180, 2063-2071. doi: 10.1128/ jb.180.8.2063-2071.1998

Murphy, K. C., Nelson, S. J., Nambi, S., Papavinasasundaram, K., Baer, C. E., and Sassetti, C. M. (2018). ORBIT: a new paradigm for genetic engineering of mycobacterial chromosomes. mBio 9:e01467-18. doi: 10.1128/mBio.01467-18

Niehaus, T. D., Thamm, A. M. K., de Crécy-Lagard, V., and Hanson, A. D. (2015). Proteins of unknown biochemical function: a persistent problem and a roadmap to help overcome it. Plant Physiol. 169, 1436-1442. doi: 10.1104/pp.15. 00959

O'Brien, M. E., Saini, A., Smith, I. E., Webb, A., Gregory, K., Mendes, R., et al. (2000). A randomized phase II study of SRL172 (Mycobacterium vaccae) combined with chemotherapy in patients with advanced inoperable non-smallcell lung cancer and mesothelioma. Br. J. Cancer 83, 853-857. doi: 10.1054/bjoc. 2000.1401

Otero, J., Jacobs, W. R., and Glickman, M. S. (2003). Efficient allelic exchange and transposon mutagenesis in Mycobacterium avium by specialized transduction. Appl. Environ. Microbiol. 69, 5039-5044. doi: 10.1128/AEM.69.9.5039-5044. 2003

Parish, T., Gordhan, B. G., McAdam, R. A., Duncan, K., Mizrahi, V., and Stoker, N. G. (1999). Production of mutants in amino acid biosynthesis genes of Mycobacterium tuberculosis by homologous recombination. Microbiology 145, 3497-3503. doi: 10.1099/00221287-145-12-3497

Parish, T., and Roberts, D. M. (2015). Mycobacteria Protocols. Totowa, NJ: Humana Press.

Parish, T., and Stoker, N. G. (2000). Use of a flexible cassette method to generate a double unmarked Mycobacterium tuberculosis tlyA plcABC mutant by gene replacement. Microbiol. Read. Engl. 146, 1969-1975. doi: 10.1099/00221287146-8-1969

Park, K. T., Dahl, J. L., Bannantine, J. P., Barletta, R. G., Ahn, J., Allen, A. J., et al. (2008). Demonstration of allelic exchange in the slow-growing bacterium Mycobacterium avium subsp. paratuberculosis, and generation of mutants with deletions at the pknG, relA, and lsr2 Loci. Appl. Environ. Microbiol. 74, 1687-1695. doi: 10.1128/AEM.01208-07

Poonam, Yennamalli, R. M., Bisht, G. S., and Shrivastava, R. (2019). Ribosomal maturation factor (RimP) is essential for survival of nontuberculous mycobacteria Mycobacterium fortuitum under in vitro acidic stress conditions. 3 Biotech 9:127. doi: 10.1007/s13205-019-1659-y

Pourcel, C., Salvignol, G., and Vergnaud, G. (2005). CRISPR elements in Yersinia pestis acquire new repeats by preferential uptake of bacteriophage DNA, and provide additional tools for evolutionary studies. Microbiol. Read. Engl. 151, 653-663. doi: 10.1099/mic.0.27437-0

Qi, L. S., Larson, M. H., Gilbert, L. A., Doudna, J. A., Weissman, J. S., Arkin, A. P., et al. (2013). Repurposing CRISPR as an RNA-guided platform for sequencespecific control of gene expression. Cell 152, 1173-1183. doi: 10.1016/j.cell.2013. 02.022

Rathnaiah, G., Bannantine, J. P., Bayles, D. O., Zinniel, D. K., Stabel, J. R., Gröhn, Y. T., et al. (2016). Analysis of Mycobacterium avium subsp. paratuberculosis mutant libraries reveals loci-dependent transposition biases and strategies for novel mutant discovery. Microbiology 162, 633-641. doi: 10.1099/mic.0. 000258

Rock, J. M., Hopkins, F. F., Chavez, A., Diallo, M., Chase, M. R., Gerrick, E. R., et al. (2017). Programmable transcriptional repression in mycobacteria using an orthogonal CRISPR interference platform. Nat. Microbiol. 2:16274. doi: 10. 1038/nmicrobiol.2016.274

Rominski, A., Selchow, P., Becker, K., Brülle, J. K., Dal Molin, M., and Sander, P. (2017). Elucidation of Mycobacterium abscessus aminoglycoside and capreomycin resistance by targeted deletion of three putative resistance genes. J. Antimicrob. Chemother. 72, 2191-2200. doi: 10.1093/jac/dkx125

Russell, D. G., Barry, C. E., and Flynn, J. L. (2010). Tuberculosis: what we don't know can, and does, hurt us. Science 328, 852-856. doi: 10.1126/science. 1184784

Rybniker, J., Wolke, M., Haefs, C., and Plum, G. (2003). Transposition of Tn5367 in Mycobacterium marinum, using a conditionally recombinant mycobacteriophage. J. Bacteriol. 185, 1745-1748. doi: 10.1128/JB.185.5.17451748.2003

Snapper, S. B., Melton, R. E., Mustafa, S., Kieser, T., and Jacobs, W. R. (1990). Isolation and characterization of efficient plasmid transformation mutants of Mycobacterium smegmatis. Mol. Microbiol. 4, 1911-1919. doi: 10.1111/j.13652958.1990.tb02040.x

Sun, B., Yang, J., Yang, S., Ye, R. D., Chen, D., and Jiang, Y. (2018). A CRISPR-Cpf1assisted non-homologous end joining genome editing system of Mycobacterium smegmatis. Biotechnol. J. 13:e1700588. doi: 10.1002/biot.201700588

Talaat, A. M., and Trucksis, M. (2000). Transformation and transposition of the genome of Mycobacterium marinum. Am. J. Vet. Res. 61, 125-128. doi: 10.2460/ ajvr.2000.61.125

Tateishi, Y., Minato, Y., Baughn, A. D., Ohnishi, H., Nishiyama, A., Ozeki, Y., et al. (2020). Genome-wide identification of essential genes in Mycobacterium intracellulare by transposon sequencing - Implication for metabolic remodeling. Sci. Rep. 10:5449. doi: 10.1038/s41598-020-62287-2

Taylor, R. K., Miller, V. L., Furlong, D. B., and Mekalanos, J. J. (1987). Use of phoA gene fusions to identify a pilus colonization factor coordinately regulated with cholera toxin. Proc. Natl. Acad. Sci. U.S.A. 84, 2833-2837. doi: 10.1073/pnas.84. 9.2833

van Kessel, J. C., and Hatfull, G. F. (2007). Recombineering in Mycobacterium tuberculosis. Nat. Methods 4, 147-152. doi: 10.1038/nmeth996

van Kessel, J. C., and Hatfull, G. F. (2008). Efficient point mutagenesis in mycobacteria using single-stranded DNA recombineering: characterization of antimycobacterial drug targets. Mol. Microbiol. 67, 1094-1107. doi: 10.1111/j. 1365-2958.2008.06109.x

van Kessel, J. C., Marinelli, L. J., and Hatfull, G. F. (2008). Recombineering mycobacteria and their phages. Nat. Rev. Microbiol. 6, 851-857. doi: 10.1038/ nrmicro2014

Vilchèze, C., and Jacobs, W. R. (2014). Resistance to Isoniazid and Ethionamide in Mycobacterium tuberculosis: genes, mutations, and causalities. Microbiol. Spectr 2, MGM2-MGM0014. doi: 10.1128/microbiolspec.MGM2-0014-2013

Viljoen, A., Gutiérrez, A. V., Dupont, C., Ghigo, E., and Kremer, L. (2018). A simple and rapid gene disruption strategy in Mycobacterium abscessus: on the design and application of glycopeptidolipid mutants. Front. Cell. Infect. Microbiol. 8:69. doi: $10.3389 /$ fcimb.2018.00069

Wards, B. J., and Collins, D. M. (1996). Electroporation at elevated temperatures substantially improves transformation efficiency of slow-growing mycobacteria. FEMS Microbiol. Lett. 145, 101-105. doi: 10.1111/j.1574-6968.1996.tb08563.x

Weerdenburg, E. M., Abdallah, A. M., Rangkuti, F., Ghany, M. A. E., Otto, T. D., Adroub, S. A., et al. (2015). Genome-wide transposon mutagenesis indicates that Mycobacterium marinum customizes its virulence mechanisms for survival and replication in different hosts. Infect. Immun. 83, 1778-1788. doi: 10.1128/ IAI.03050- 14

Whittington, R., Donat, K., Weber, M. F., Kelton, D., Nielsen, S. S., Eisenberg, S., et al. (2019). Control of paratuberculosis: who, why and how. A review of 48 countries. BMC Vet. Res. 15:198. doi: 10.1186/s12917-019-1943-4

Wilson, T., Wards, B. J., White, S. J., Skou, B., de Lisle, G. W., and Collins, D. M. (1997). Production of avirulent Mycobacterium bovis strains by illegitimate recombination with deoxyribonucleic acid fragments containing an interrupted ahpC gene. Tuber. Lung Dis. Off. J. Int. Union Tuberc. Lung Dis. 78, 229-235. doi: 10.1016/s0962-8479(97)90003-4

Wu, M.-L., Aziz, D. B., Dartois, V., and Dick, T. (2018). NTM drug discovery: status, gaps and the way forward. Drug Discov. Today 23, 1502-1519. doi: 10.1016/j.drudis.2018.04.001

Yan, M.-Y., Li, S.-S., Ding, X.-Y., Guo, X.-P., Jin, Q., and Sun, Y.-C. (2020). A CRISPR-assisted nonhomologous end-joining strategy for efficient genome 
editing in Mycobacterium tuberculosis. mBio 11:e02364-19. doi: 10.1128/mBio. 02364-19

Yan, M.-Y., Yan, H.-Q., Ren, G.-X., Zhao, J.-P., Guo, X.-P., and Sun, Y.-C. (2017). CRISPR-Cas12a-assisted recombineering in bacteria. Appl. Environ. Microbiol. 83:e0947-17. doi: 10.1128/AEM.00 947-17

Yu, D., Ellis, H. M., Lee, E.-C., Jenkins, N. A., Copeland, N. G., and Court, D. L. (2000). An efficient recombination system for chromosome engineering in Escherichia coli. Proc. Natl. Acad. Sci. U.S.A. 97, 5978-5983. doi: 10.1073/pnas. 100127597

Zhang, Q., Wang, D., Jiang, G., Liu, W., Deng, Q., Li, X., et al. (2016). EsxA membrane-permeabilizing activity plays a key role in mycobacterial cytosolic translocation and virulence: effects of single-residue mutations at glutamine 5 . Sci. Rep. 6:32618. doi: 10.1038/srep32618
Zhang, Y., Buchholz, F., Muyrers, J. P., and Stewart, A. F. (1998). A new logic for DNA engineering using recombination in Escherichia coli. Nat. Genet. 20, 123-128. doi: 10.1038/2417

Conflict of Interest: The authors declare that the research was conducted in the absence of any commercial or financial relationships that could be construed as a potential conflict of interest.

Copyright (c) 2021 Chimukuche and Williams. This is an open-access article distributed under the terms of the Creative Commons Attribution License (CC BY). The use, distribution or reproduction in other forums is permitted, provided the original author(s) and the copyright owner(s) are credited and that the original publication in this journal is cited, in accordance with accepted academic practice. No use, distribution or reproduction is permitted which does not comply with these terms. 